\title{
Proposta de uma equação de regressão para estimar o pico da frequencia cardíaca ou a frequencia cardíaca máxima de esforço em indoor cycling
}

Leandro Abreu Pereira

Rui Manuel Garganta

Faculdade de Desporto, Universidade do Porto, Porto, Portugal

Pereira, L.; Garganta, R.; Proposta de uma equação de regressão para estimar o pico da frequencia cardíaca ou a frequencia cardíaca máxima de esforço em indoor cycling Motricidade 3(2): 81-87

\section{Resumo}

Introdução: uma das modalidades de academia que maior sucesso tem conquistado é o Indoor Cycle (IC), no entanto, o controlo da intensidade do esforço nem sempre é realizado e quando o é, recorre a uma fórmula universal que pode ter subjacente um erro grosseiro. Objectivos: (1) sugerir uma equação para determinar a frequência cardíaca máxima de esforço $\left(\mathrm{FC}_{\text {máx }} \mathrm{E}\right)$ ou Pico de FC passível de ser aplicada nas aulas de IC e (2) comparar a fórmula por nós sugerida com as propostas pelo ACSM (2006), Fox et al (1991) Tanaka (2001). Metodologia: a amostra é constituída por 135 adultos (33 \pm 9 anos), praticantes de Indoor Cycling, há pelo menos 6 meses e aparentemente saudáveis segundo os critérios do ACSM (2002). Durante a aula foi sugerido um protocolo de avaliação da $\mathrm{FC}_{\text {máx }} \mathrm{E}$, apresentado por Garganta e Roig (2005). Os dados foram recolhidos com um Polar Team System ${ }^{\mathrm{TM}}$, nos ginásios "Stress Out", "Paços Health Club", "Bfree Fitness Club" e "Academia Impacto". A equação de previsão foi elaborada com base na regressão linear simples, para um nível de significância de 0.05 . Resultados: de acordo com a análise aos coeficientes de regressão, a fórmula que sugerimos é a seguinte: $\mathrm{FC}_{\text {máx }} \mathrm{E}_{(\mathrm{IC})}=205-0,7 \star$ Idade, $\left(\mathrm{r}=0,61, \mathrm{r}^{2}=0,36\right.$, epe $\left.=7,5 \mathrm{bpm}\right)$. Discussão: tal como vem referenciado na literatura, as equações de regressão devem ser entendidas como especificas e não universais, visto que há diferenças significativas entre a fórmula estimada durante as aulas de Indoor Cycle e as habitualmente sugeridas na literatura.

Palavras-Chave: Cárdio-vascular; Frequência cardíaca; Indoor Cycle

\section{Abstract}

Proposition of a regression equation of peak heart rate for indoor cycling

Background: one of the most successful academy modalities is the Indoor Cycle (IC), however, the effort control intensity isn't always carried through, and when it is, it appeals to a universal formula that might have underlying a gross error. Objectives: (1) suggesting an equation to determine the Peak of FC or FC max E to be applied in the classes of IC and (2) comparing the formula suggested by us together with the ACSM (2006) propositions, Fox et al (1991) Tanaka (2001). Methodology: the sample is constituted by 135 adults (33 \pm 9 years), who practise Indoor Cycling, for about 6 months and apparently healthy according to ACSM (2002) criterie. During a class, it was suggested a protocol of evaluation of the peak of $\mathrm{FC}$ or $\mathrm{FC}_{\max } \mathrm{E}$, suggested by Garganta and Roig (2005). The data were collected with a Polar Team System TM, in the "Stress Out", "Paços Health Club", "Bfree Fitness Club" and "Academy Impact" academies. Results: according to the analysis to the regression coefficients, the following equation come up: $\mathrm{FC}_{\max } \mathrm{E}(\mathrm{IC})=205-0,7 \star$ Idade, $(\mathrm{r}=0,61, \mathrm{r} 2=0,36$, epe $=7,5 \mathrm{bpm})$. Discussion: we verified that there are significant differences between the estimated formula during the Indoor Cycle classes and currently and used formulas and the regression equations must be understood as you specify and not universal.

Key-words: Cardio-vascular; Heart Rate; Indoor Cycle 


\section{Introdução}

O exercício físico tem-se constituído como um dos meios fundamentais para a promoção da saúde e bem-estar das populações. As possibilidades para a sua aplicação são cada vez mais variadas, sobretudo nos ginásios, onde o número de propostas de exercitação tem vindo a aumentar de forma significativa. Dentro destas, o treino cárdio-vascular tem vindo a assumir um protagonismo importante e, de entre as diferentes propostas, as actividades que o contemplam assumem-se como maioritárias. Uma das mais paradigmáticas, pelo sucesso que vem conquistando, é o Indoor Cycle (IC) que, a seguir ao STEP se vem assumindo como a modalidade com maior perspectiva de êxito. Todavia, tal como as restantes "aulas de grupo", o IC não possui grande vocação para responder às exigências, capacidades e necessidades dos diferentes praticantes, sendo a intensidade de esforço, habitualmente, determinada pelo professor em função do ritmo musical $(\mathrm{bpm})$ "imposto" e não relativamente às capacidades e objectivos dos praticantes ${ }^{6,7}$. Para além disso, a intensidade do esforço, quando avaliada, tem sido comparada com equações universais, que foram estimadas em testes laboratoriais $2,5,8,12$ ou por metanálise ${ }^{13}$, isto é, fora do contexto real da modalidade. Curiosamente a fórmula apresentada pela instituição de referência ${ }^{4}$, no que se refere à prescrição de exercício cárdio-vascular é: $\mathrm{FC}_{\max }=220$-idade. Tal fórmula é, no mínimo controversa, atendendo a que tem um erro muito elevado e não tem em consideração o tipo de exercício, quando se sabe que as estimativas deste género são específicas do modo de exercício e da população onde foram desenvolvidas. Assim, Fox et al. ${ }^{5}$, sugeriram a seguinte equação: $\mathrm{FC}_{\max }$ $=215.4-0.9147$ (idade), que apresenta como :ึّ. valores de correlação $(\mathrm{r}=0.51)$, coeficiente de determinação $\left(r^{2}=0.26\right)$ e erro padrão de estimativa $(21 \mathrm{bpm})$. Mais recentemente, Tanaka ${ }^{13}$ apre$\underset{\infty}{\infty}$ sentou uma equação que resultou de um estudo de metanálise: $\mathrm{FC}_{\text {máx }}=208.754-0.734 \star$ (idade). Relativamente à proposta por Fox et al. ${ }^{5}$, revela valores mais elevados de correlação $(r=0.93)$, maior coeficiente de determinação $\left(r^{2}=0.86\right)$ e um erro padrão de estimativa menor ( $7 \mathrm{bpm})$. De referir ainda, que todas enfermam do mesmo erro: não são específicas de nenhum modo de exercício mas universais.

Outro aspecto importante, relaciona-se com a dificuldade em avaliar a $\mathrm{FC}_{\text {maxx }}$ de acordo com os critérios estabelecidos pela literatura, visto que exige patamares de intensidade crescentes (nos ergómetros de IC não podemos ter a noção real da carga) e, tempos bem definidos para cada patamar (um mínimo de 3 min. para que a FC estabilize). Mais uma vez, podemos constatar que a sua avaliação no contexto ecológico não pode ser realizada. Por este motivo pensamos que o indicador que deve servir de base para a prescrição de exercício nesta modalidade é a $\mathrm{FC}_{\text {máx }}$ de Esforço $\left(\mathrm{FC}_{\text {máx }} \mathrm{E}\right)$ ou Pico de FC.

Assim, o presente trabalho tem dois objectivos: (1) sugerir uma equação para determinar a $\mathrm{FC}_{\max }$, passível de ser aplicada nas aulas de IC; (2) comparar a fórmula por nós sugerida com as propostas pelo ACSM ${ }^{4}$, Fox et $\mathrm{al}^{5} \mathrm{e}^{\text {Tanaka }}{ }^{13}$.

\section{Metodologia}

\section{Amostra}

A nossa amostra (tabela 1), é constituída por 135 sujeitos de ambos os sexos (75 homens e 60 mulheres). 
Proposta de uma equação de regressão para estimar o pico da frequencia cardíaca ou a frequencia cardíaca máxima de esforço em indoor cycling

Leandro Abreu Pereira e Rui Manuel Garganta

\begin{tabular}{lcc}
\hline \multicolumn{2}{l}{ Tabela 1: Caracterização da amostra } & \\
\hline Variáveis Observadas & Média $\pm \mathrm{Dp}$ & Amplitude \\
Idade (anos) & $32 \pm 8$ & $19-55$ \\
$\mathrm{FC}_{\text {máx }} \mathrm{E}(\mathrm{bpm})$ & $183 \pm 9$ & $150-200$ \\
Peso (Kg) & $68,7 \pm 12,0$ & $46-98$ \\
\hline
\end{tabular}

Os critérios de inclusão na amostra do nosso estudo foram: (1) ser praticante da modalidade à pelo menos 6 meses; (2) ser aparentemente saudável de acordo com os critérios do ACSM 3; (3) não possuir problemas de coluna; (4) estar enquadrado numa faixa etária dos 18 aos 60 anos. Após descrição detalhada dos objectivos e procedimentos inerentes às avaliações a realizar, todos os sujeitos seleccionados, assinaram um documento em que aceitavam participar no estudo.

\section{Procedimentos}

A recolha dos dados foi efectuada entre Janeiro e Abril de 2005 no ginásio "Stress Out" e durante Setembro e Outubro de 2006 nos ginásios "Paços Health Club", "Bfree Fitness Club" e "Academia Impacto". As aulas têm uma duração aproximada de 45 minutos, dividida em quatro segmentos: aquecimento (cerca de 5 minutos), parte fundamental (35 minutos), alongamentos (cerca de 2 a 3 minutos) e relaxamento (cerca de 2 a 3 minutos). O ritmo de pedalada (rpm) foi de: 135 no aquecimento, 125 a 145 na fase fundamental e sem ritmo definido nos alongamentos e relaxamento. Procuramos ainda, que o pico da FC ${ }^{10}$ fosse atingida durante os últimos 10 minutos da parte fundamental, sendo sugerido durante esse tempo, um incremento de carga de minuto a minuto através de um manípulo incorporado na bicicleta, de forma a aumentar a resistência da pedalada (intensidade). A tabela 2 demonstra a carga sugerida (de acordo com a sensação subjectiva de esforço) durante o teste.

\section{Estatística}

Para a interpretação dos resultados recorremos aos valores da estatística descritiva média e desvio padrão. A normalidade da distribuição foi confirmada pelo teste de Kolmogorov Smimov. A equação de predição para a $\mathrm{FC}_{\text {máx }} \mathrm{E}$ em Indoor Cycle foi realizada com base na análise de regressão linear. Para comparar as fórmulas que predizem a $\mathrm{FC}_{\text {máx }}$ com a nossa proposta, utilizamos a ANOVA de Medidas Repetidas com o

Tabela 2: Carga/sensação subjectiva de esforço sugerida ao longo dos 10 minutos de teste Minutos Carga/sensação Subjectiva de Esforço

\begin{tabular}{|c|c|}
\hline 1 & Leve \\
\hline 2 & Leve \\
\hline 3 & Moderada \\
\hline 4 & Moderada \\
\hline 5 & Forte \\
\hline 6 & Forte \\
\hline 7 & Muito Forte \\
\hline 8 & Muito Forte \\
\hline 9 & Muito Forte \\
\hline 10 & Extremamente Forte \\
\hline
\end{tabular}




\begin{tabular}{|c|c|c|c|c|c|}
\hline \multirow[t]{2}{*}{ Modelo } & \multicolumn{2}{|c|}{$\begin{array}{l}\text { Coeficientes não } \\
\text { estandardizados }\end{array}$} & \multirow{2}{*}{$\begin{array}{c}\text { Coeficientes } \\
\text { estandardizados } \\
\text { Beta }\end{array}$} & \multirow[t]{2}{*}{$\mathrm{t}$} & \multirow[t]{2}{*}{ Sig. } \\
\hline & B & $\mathrm{Dp}$ & & & \\
\hline Sexo & 3,01 & 3,12 & 14 & .97 & ,340 \\
\hline Idade &,- 81 & 10 &,- 65 & $-7,81$ & ,001 \\
\hline Peso &,- 11 & 12 &,- 13 &,- 92 &, 360 \\
\hline Tempo de prática &,- 06 & ,08 & ,08 &, 79 & ,431 \\
\hline Frequência Semanal &,- 33 & 1,12 &,- 03 &,- 29 &, 770 \\
\hline $\mathrm{FC}_{\text {rep }}$ & 10 & ,05 & 17 & 1,99 & ,054 \\
\hline
\end{tabular}

teste Bonferroni para as múltiplas comparações. O nível de significância utilizado foi de 0,05 . Os resultados foram tratados em SPSS 14.0.

\section{Resultados}

Numa primeira fase utilizamos 5 indicadores, para predizer a $\mathrm{FC}_{\text {máx }} \mathrm{E}$. Todavia, tal como podemos ver na tabela 3 , apenas a idade apresentou uma regressão com significado estatístico.

Assim sendo, utilizamos a idade como o único pedictor, tal como podemos constatar da tabela 4 . Assim sendo, através dos coeficientes de regressão surge a seguinte a equação: $\mathrm{FC}_{\text {maxx }} \mathrm{E}=205-0.7$ (Idade), com: $\mathrm{r}=0,61, \mathrm{r}^{2}=0,36$ e epe $=7,5 \mathrm{bpm}$.

Após termos encontrado chegado a nossa equação final, efectuamos uma comparação entre esta e as restantes equações universais, e verificamos que a equação de Indoor Cycling apresenta valores de correlação mais elevados que a sugerida por Fox e mais baixos que a de Tanaka. Consequentemente, temos um erro padrão de estimativa menor que a de Fox e idêntico ao da fórmula proposta por Tanaka tal como se pode verificar através da tabela 5 . Não há sugestões para a fórmula do ACSM.

De forma, a podermos confirmar se é indiferente utilizar a fórmula sugerida pelo nosso estudo ou as apresentadas pela literatura $4,5,8,11,13,14,15$, real-

\begin{tabular}{lccccc}
\hline \multicolumn{5}{c}{ Tabela 4: Coeficientes de regressão e níveis de significância para a variável predictora. } \\
\hline \multicolumn{7}{c}{ Coeficientes não } & Coeficientes & & \\
Modelo & estandardizados & estandardizados & t & Sig. \\
& $B$ & Dp & Beta & & \\
$\mathrm{FC}_{\text {máx }}$ (Constante) & 204,7 & 2,6 & & 79,46 &, 00 \\
Idade &,- 7 &, 08 &,- 61 & $-8,81$ &, 00 \\
\hline
\end{tabular}

\begin{tabular}{|c|c|c|c|c|}
\hline Equações & Fórmula & $\mathrm{R}$ & $\mathrm{R}^{2}$ & Epe \\
\hline IC & $\mathrm{FC}_{\text {máx }} \mathrm{E}=205-0,7 *$ idade & 0,61 & 0,37 & 7,5 \\
\hline ACSM & $\mathrm{FC}_{\text {máx }}=220$-idade & $?$ & $?$ & $?$ \\
\hline Fox & $\mathrm{FC}_{\text {máx }}=215.4-0.9147 *$ idade & 0,51 & 0,26 & 21 \\
\hline Tanaka & $\mathrm{FC}_{\text {máx }^{*}}=208.754-0.734 *$ idade & 0,93 & 0,86 & 7 \\
\hline
\end{tabular}


Proposta de uma equação de regressão para estimar o pico da frequencia cardíaca ou a frequencia cardíaca máxima de esforço em indoor cycling

Leandro Abreu Pereira e Rui Manuel Garganta

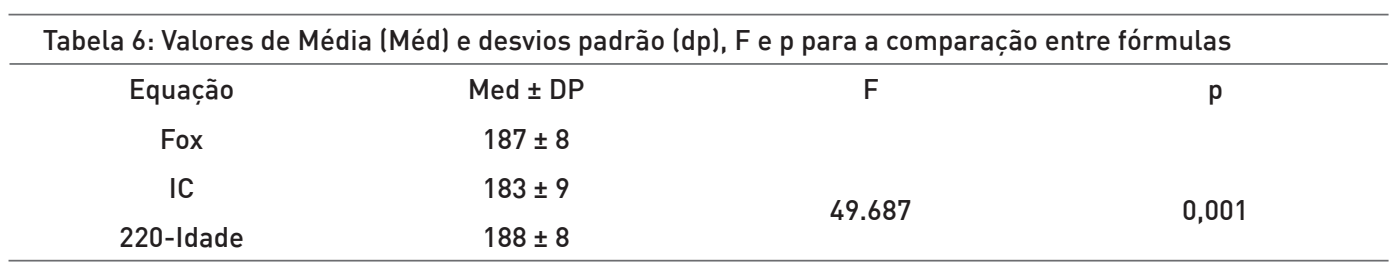

izamos uma ANOVA de medidas repetidas, e os resultados $(F=49,687 ; \mathrm{p}=0.001)$ sugerem que as equações providenciam uma informação diferente da proposta por nós (tabela 6). significativas entre a fórmula estimada durante as aulas de Indoor Cycle e as habitualmente sugeridas na literatura e (2) as equações de regressão devem ser entendidas como especificas e não universais.

\begin{tabular}{lccc}
\hline \multicolumn{2}{c}{ Tabela 7: Valores do teste de Bonferroni para as diferentes equações } & \\
\hline Equações & Dif. de médias & Amplitude & $\mathrm{p}$ \\
IC vs (220-idade) & 6 & $0-26$ & 0.001 \\
IC vs Tanaka & 3 & $0-20$ & 0.001 \\
IC vs Fox & 4 & $0-23$ & 0.001 \\
\hline
\end{tabular}

Posteriormente, efectuámos o teste de múltiplas comparações (Bonferroni) (tabela 7), o que nos permitiu constatar havia diferenças estatisticamente significativas entre todas as fórmulas.

\section{Discussão}

Os resultados sugerem que a selecção da fórmula para a predição da $\mathrm{FC}_{\text {máx }} \mathrm{E}$ deve ser efectuada de um forma cuidadosa e ponderada. Assim sendo, não é indistinto recorrer a qualquer uma das equações para a prática do Indoor Cycling, dado que as diversas equações ${ }^{1,2,3,4,5,13}$ apresentam valores substancialmente distintos, o que seguramente implicará respostas fisiológicas diferenciadas.

Importa ainda realçar que temos a noção de que a situação ideal para o cálculo da $\mathrm{FC}_{\text {máx }} \mathrm{E}$, é realizar um teste máximal no seu contexto "ecológico". Todavia, devido às dificuldades existentes relacionadas com a falta de material nos ginásios, à impossibilidade de realizar testes máximos em determinados alunos e assumindo o factor de risco associado a um teste desta natureza, sugerimos uma equação que se constitui como uma alternativa de recurso e que é específica da modalidade em causa.

Podemos ainda, concluir que (1) há diferenças

\section{Correspondência}


Leandro Abreu Pereira,

Rua Padre António Vieira, 2º ${ }^{\circ}$ n 32, 4425

-702 ,

Pedrouços.

Leandro_acores@hotmail.com Referências

1. American College of Sports Medicine (1990). The Recommended quantity and quality of exercise for developing and maintaining cardiorespiratory and muscular fitness in health adults. Med Sci Sports Exercise.

2.American College of Sports Medicine (1995). Guidelines for exercise testing and prescription (5 ed.). Philadelphia:Williams \& Wilkins.

3.American College of Sports Medicine (2002). Guidelines for exercise testing and prescription (5 ed.). Philadelphia:Williams \& Wilkins.

4.American College of Sports Medicine . (2006). Guidelines for exercise testing and prescription. (7 ed.). Philadelphia: Lippincott Williams \& Wilkins.

5. Fox E. (1991). Bases fisiológicas da educação física e dos desportos. Rio de Janeiro: Guanabara Koogan.

6. Garganta R. (2000). Caracterização do esforço e efeitos induzidos pela prática de actividades de academia na Aptidão Física e no Auto-conceito Físico: Estudo realizado em adultos jovens do sexo feminino praticantes de Ginástica Aeróbica, Musculação e Cardiofitness. Dissertação de Doutoramento. Porto: Faculdade de Ciências do Desporto e Educação Física da Universidade do Porto.

7. Garganta R. (2005) Guia Prático de Avaliação Física em Ginásios, Academias e Health Clubs. 灾 Cacém: Manz Produções.

\% 8. McArdle WD; Katch FI; Katch V. (1998). Fisiologia do exercício: Energia, Nutrição e $\lesssim$ Desempenho Humano. (4 ed) Rio de Janeiro:
Guanabara Koogan..

9. Pollock ML, Wilmore JH. (1993) Exercícios na saúde e na doença. Avaliação e prescrição para prevenção e reabilitação. (2 ed) Rio de Janeiro: MEDSI.

10. Rasoilo J. (1998). Utilização de monitores de FC no controle do treino. Revista Treino Desportivo. 1(5):39-44.

11. Robergs R; Roberts S. (1996). Exercise Physiology:exercise performance and clinical applications. St. Louis: Missouri:Mosby.

12. Roig J, Garganta R (2005). Caracterização do esforço e elaboração de uma estratégia para a determinação de zonas alvo de treino em R P M. Lisboa: Manz Produções.

13. Tanaka H. (2001). Age-predicted maximal heart rate revisited. J Am Col Card. 37 (1):153156.

14. Wilmore J; Costill D. (1998). Fisiologia del esfuerzo y del deporte.Barcelona: Paidotribo.

15. Wilmore J; Costill D. (1999). Physiology of sport and exercise (2 ed.). Champaign: Human Kinetics Publishers. 